\title{
A time-selective technique for free-field reciprocity calibration of condenser microphones
}

Barrera Figueroa, Salvador; Rasmussen, Knud; Jacobsen, Finn

Published in:

Acoustical Society of America. Journal

Link to article, DOI:

$10.1121 / 1.1604123$

Publication date:

2003

Document Version

Publisher's PDF, also known as Version of record

Link back to DTU Orbit

Citation (APA):

Barrera Figueroa, S., Rasmussen, K., \& Jacobsen, F. (2003). A time-selective technique for free-field reciprocity calibration of condenser microphones. Acoustical Society of America. Journal, 114(3), 1467-1476.

https://doi.org/10.1121/1.1604123

\section{General rights}

Copyright and moral rights for the publications made accessible in the public portal are retained by the authors and/or other copyright owners and it is a condition of accessing publications that users recognise and abide by the legal requirements associated with these rights.

- Users may download and print one copy of any publication from the public portal for the purpose of private study or research.

- You may not further distribute the material or use it for any profit-making activity or commercial gain

- You may freely distribute the URL identifying the publication in the public portal

If you believe that this document breaches copyright please contact us providing details, and we will remove access to the work immediately and investigate your claim. 


\title{
A time-selective technique for free-field reciprocity calibration of condenser microphones
}

\author{
Salvador Barrera-Figueroa, ${ }^{\text {a) }}$ Knud Rasmussen, ${ }^{\text {b) }}$ and Finn Jacobsen ${ }^{c)}$ \\ Technical University of Denmark, Ørsted-DTU, Acoustic Technology, Ørsted Plads, Building 352, \\ DK-2800 Lyngby, Denmark
}

(Received 12 September 2002; revised 6 June 2003; accepted 8 July 2003)

\begin{abstract}
In normal practice, microphones are calibrated in a closed coupler where the sound pressure is uniformly distributed over the diaphragm. Alternatively, microphones can be placed in a free field, although in that case the distribution of sound pressure over the diaphragm will change as a result of the diffraction of the body of the microphone, and thus, its sensitivity will change. In the two cases, a technique based on the reciprocity theorem can be applied for obtaining the absolute sensitivity either under uniform pressure or free-field conditions. In this paper, signal-processing techniques are considered that improve the accuracy of the free-field calibration method. In particular, a fast Fourier transform (FFT)-based time-selective technique for removing undesired reflections from the walls of the measurement chamber has been developed and applied to the electric transfer impedance function between two microphones. The acoustic centers of the microphones have been determined from the "cleaned" transfer impedance values. Then, the complex free-field sensitivities of the microphones have been calculated. The resulting complex sensitivities and acoustic centers have proved to be in good agreement with previously published data, and this confirms the reliability of the time-selective technique, even in nonanechoic environments. Furthermore, the obtained results give a new reference for further comparisons, because they cover a frequency range with an accuracy that has not been obtained by previously published data. (C) 2003 Acoustical Society of America. [DOI: 10.1121/1.1604123]
\end{abstract}

PACS numbers: 43.38.Kb, 43.20.Ye, 43.60.Qv [DKW]

\section{INTRODUCTION}

The absolute determination of the free-field sensitivity of a condenser microphone is carried out making use of the reciprocity technique. The free-field reciprocity calibration of microphones was first discussed by MacLean. ${ }^{1}$ Later, Wathen-Dunn ${ }^{2}$ described in more detail some theoretical aspects of the calibration, such as the acoustic center of the microphones, and suggested that the coupling of the microphones may occur. Rudnick and Stein ${ }^{3}$ presented an experimental study of the free-field calibration based on the works of MacLean and DiMattia. ${ }^{4}$ Further experimental attempts of free-field calibrations were described by Terry $^{5}$ and Niemoller, ${ }^{6}$ who introduced the possibility of carrying out calibrations in the time domain. In the 1990's, some national metrology institutes described the experimental apparatus used for free-field reciprocity calibrations, and presented some results. ${ }^{7-11}$ Finally, the calibration method has been standardized. ${ }^{12}$

In the implementation of the free-field calibration setup a number of problems occur. The first is a very poor signalto-noise ratio that can be negative at low frequencies when measuring the electrical transfer impedance between the two microphones. This can be partially solved by applying some signal conditioning techniques and by measuring the electrical transfer impedance at short distances between the microphones. However, it is worth mentioning that when the dis-

\footnotetext{
${ }^{a)}$ Electronic mail: sbf@oersted.dtu.dk

${ }^{b)}$ Electronic mail: kr@oersted.dtu.dk

${ }^{c)}$ Electronic mail: fja@oersted.dtu.dk
}

tance between the microphones is sufficiently short, a standing wave could be formed between the diaphragms of the microphones.

Another problem present in the free-field calibration is the nonideal performance of the anechoic environments developed to realize the free field under controlled environmental conditions. The problem is due to reflections from the walls of the anechoic chamber. These reflections and the standing wave between the microphones, though small, can have a significant influence on the measured electrical transfer impedance function between the microphones, and thus on the free-field sensitivity of the microphones. If a low uncertainty on the determined value of the sensitivity is to be achieved, the effect of such reflections must be reduced or eliminated from the electrical transfer impedance between the microphones. This can be determined in the time domain by means of a gating or time-selective technique either in real time or by postprocessing the data obtained in the frequency domain. Earlier work that includes time-selective techniques on the free-field calibration of condenser microphones has been done by Lambert and Durocher, ${ }^{13}$ and Blem, ${ }^{14}$ who explored the possibility of removing unwanted reflections by applying a time-selective window using an FFT-based procedure. Vorländer ${ }^{15}$ also proposed the application of a time-selective window to the impulse response, which was obtained by the use of broadband signals. In this paper, an FFT-based time-selective technique is analyzed.

The proposed procedure consists of three major stages: (a) a treatment of the frequency response; (b) the determina- 


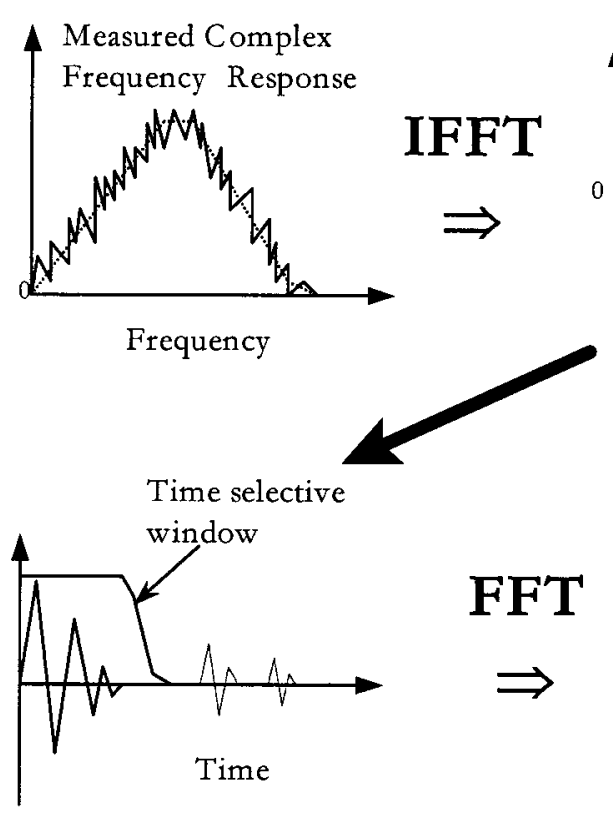

Direct Impulse

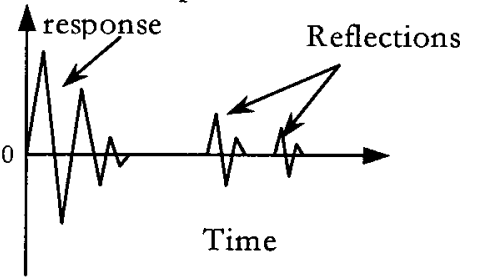

FIG. 1. Schematic of the procedure for removal of reflections of a complex frequency response function.

tion of the impulse response and the application of a timeselective window; and (c) the calculation of the cleaned frequency response.

It is possible to measure the electrical transfer impedance at equidistantly placed frequencies in a finite frequency range. If any Fourier transformation is to be applied, the missing portions of the frequency response must be completed by an adequate procedure. Then, the inverse Fourier transform is applied to the completed frequency response in order to obtain the impulse response. It contains information about the direct wave between the microphones as well as the reflections and standing waves. These undesired effects can be removed by means of a time-selective window.

Finally, the cleaned electrical transfer impedance can be obtained by applying the Fourier transform. This result can be used for determining the acoustic centers of the microphones and the free-field sensitivity of the condenser microphone. A schematic representation of the procedure is shown in Fig. 1.

The overall effect of the application of the cleaning procedure over the accuracy of the microphone sensitivity is studied by making use of a simulated transfer impedance between the two microphones. These results are used for validating the application of the time-selective technique by comparing them with complementary experimental and numerical results.

\section{THE ELECTRICAL TRANSFER IMPEDANCE}

Consider two microphones located at an acoustical distance $d_{12}$ from each other in a free field. The electrical transfer impedance between the microphones at the frequency $f$ is defined in Ref. 12 as the ratio of the open-circuit voltage on the electrical terminals of the receiver microphone, $u_{2}$, to the electrical current through the electrical terminals of the second microphone acting as sound source, $i_{1}$

$$
Z_{e, 12}=\frac{u_{2}}{i_{1}}=j \frac{\rho f}{2 d_{12}} M_{f, 1} M_{f, 2} \exp \left(-j \gamma d_{12}\right)
$$

where $M_{f, 1}$ and $M_{f, 2}$ are the free-field sensitivities of the microphones, $\rho$ is the density of the medium, and $\gamma$ is the complex propagation coefficient, which includes the air absorption. The electrical transfer impedance is the basis of the reciprocity technique as it contains information about the free-field sensitivities of the two microphones, thus providing means for determining their sensitivities. Figure 2 shows a diagram of the microphones in the free field.

Information regarding the shape of the corresponding impulse response can be extracted from the factors in Eq. (1). It is possible to divide the electrical transfer impedance into three different factors. The first is the frequency multiplied by a constant, the second is the product of sensitivities, and the third is the complex exponential of the product of acoustical distance and the complex propagation coefficient. The

\section{Homogeneous, unbounded medium $\rho, c$}

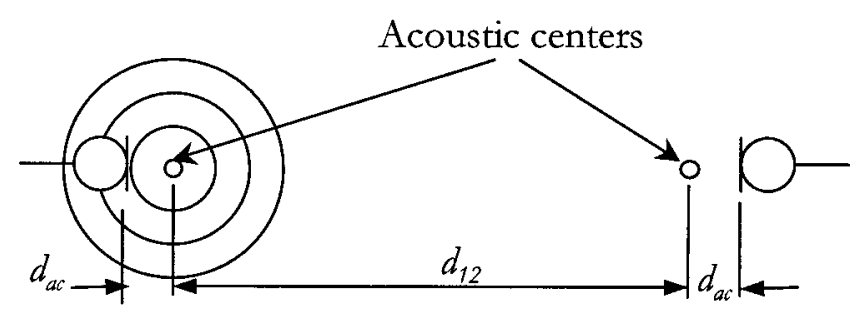

$$
\begin{aligned}
& \text { Transmitter } \\
& \text { microphone }
\end{aligned}
$$

\section{Receiver microphone}

FIG. 2. Schematic of two microphones in a free field. One of the microphones is acting as a sound source (transmitter) and the other as a receiver. 


\section{Frequency Domain}
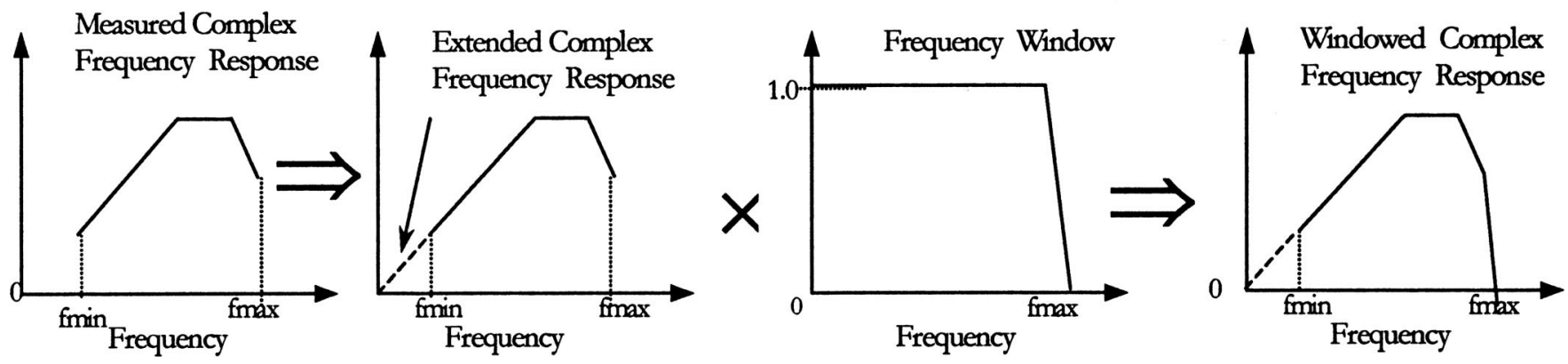

FIG. 3. Schematic of the procedure for the treatment of an incomplete frequency response prior to the Fourier analysis.

shape of the impulse response of the electrical transfer impedance is the convolution of the impulse responses of each factor.

The first term may be considered as a differentiation that emphasizes high frequencies; this is a consequence of the fact that the transmitter microphone is acting as a monopole. The second term can be considered as a multiplication of two systems, each of a single degree of freedom. The last term can be interpreted as a time delay. This implies that the variations observed in the impulse response should be mostly associated with the variations in the parameters of the two single-degree-of-freedom systems associated with the freefield sensitivities. This indicates that the parameters of the time-selective procedure should be selected taking such information as a basis.

A quantity used for determining the time it takes the impulse response of a system of a single degree of freedom to decay to an arbitrary level with respect to its maximum amplitude is the time constant, $\tau$, which is given by

$$
\tau=\frac{2 m_{a}}{R_{a}},
$$

where $m_{a}$ is the acoustical mass and $R_{a}$ is the acoustical resistance from the system of a single degree of freedom.

\section{REMOVAL OF REFLECTIONS}

The reflections from the walls of the anechoic chamber and standing waves between the microphones can be eliminated from the electrical transfer impedance, $Z_{e, 12}(f)$, using the following procedure. The impulse response of the electrical transfer impedance, $z_{e, 12}(t)$, can be calculated by applying the inverse Fourier transform (IFT). In the time domain, the reflections appear as scaled and perhaps distorted replicas delayed from the main impulse response. Thus a time-selective window, $w(t)$, may be applied to remove them. Then, the "cleaned" electrical transfer impedance, $Z_{e, 12, C}(f)$, can be calculated by applying the Fourier transform to the windowed impulse response, $z_{e, 12, w}(t)$. The procedure graphically shown in Fig. 1 can be formalized as

$Z_{e, 12}(f) \stackrel{\text { IDFT }}{\longrightarrow} z_{e, 12}(t) \stackrel{w(t)}{\longrightarrow} z_{e, 12, w}(t) \stackrel{\text { DFT }}{\longrightarrow} Z_{e, 12, C}(f)$.

According to the definition of the IFT of a complex frequency response, $X(f)$

$$
x(t)=\int_{-\infty}^{\infty} X(f) \exp (j 2 \pi f t) d f,
$$

the frequency response must be known at all frequencies. However, in practice, it is only possible to measure the electrical transfer impedance in a limited frequency range. There is a low-frequency limit, $f_{0}$, below which it is impossible to measure the electrical transfer impedance. This is caused by the microphone's thermal noise. ${ }^{16}$ When combined with the microphone's very low radiation capabilities, this results in an extremely poor signal-to-noise ratio at low frequencies. On the high-frequency side, the limitations come from the capacity of the data acquisition software, which makes it possible to measure only up to a frequency $f_{\max }$ where the electrical transfer impedance may not have decayed sufficiently.

If any Fourier transform-based postprocessing is to be performed on the electrical transfer impedance, it has to be defined in the whole frequency interval, formally from $-\infty$ to $+\infty$ as in Eq. (4), or from 0 to $+\infty$ for a one-sided frequency response. Then, a treatment of the missing portions of the frequency response must be carried out.

\section{A. Treatment of the frequency response}

The frequency response must be completed in the whole frequency domain before the inverse Fourier transform is applied. This means that the low-frequency portion $\left[0, f_{0}\right]$ and the high-frequency portion $\left[f_{\max },+\infty\right]$ must be generated in such a way that follows the physical behavior of the function without modifying the measured electrical transfer impedance function. The procedure is shown schematically in Fig. 3.

\section{Low-frequency artificial extension of the electrical transfer impedance}

The missing low-frequency values can be completed by patching ideal values to the measured of the electrical transfer impedance. This solution is based on the fact that at low frequencies, diffraction and radiation impedance effects are almost negligible, and the free-field sensitivity becomes constant and almost equal to the pressure sensitivity. Thus, a lumped-parameter model can be used for generating the ideal electrical transfer impedance. ${ }^{17}$ The lumped parameters can be determined from pressure sensitivity data. The resulting patched electrical transfer impedance, $Z_{e, 12, E}(f)$, is defined as 


$$
Z_{e, 12, E}(f)=\left\{\begin{array}{ll}
Z_{e, 12, i}(f) & 0 \leqslant f \leqslant f_{0} \\
Z_{e, 12, m}(f) & f_{0} \leqslant f \leqslant f_{\max }
\end{array},\right.
$$

where $Z_{e, 12, i}$ is the ideal electrical transfer impedance calculated from the lumped-parameter model of the microphone and $Z_{e, 12, m}(f)$ is the measured electrical transfer impedance.

One of the possible shortcomings of this procedure is that the effect of the reflections is larger at low frequencies. As the procedure uses an ideal, reflectionless frequency response for filling the missing part, a realistic representation of the disturbances may not be obtained, though ultimately the reflections are to be removed. Furthermore, the patching of ideal data to measured data may introduce an additional problem. The value of the electrical transfer impedance at the lowest measured frequency, $f_{0}$, may correspond either to a maximum or a minimum of the standing wave pattern. This will introduce a discontinuity on the slope of the extended electrical transfer impedance function that may be reflected onto the impulse response.

\section{Low-pass filtering of the electrical transfer impedance}

The sensitivity of a microphone tends to decay at high frequencies as a consequence of the mechanical behavior of the diaphragm. This is explained by the fact that above the resonance frequency of the diaphragm, its movement is controlled by its mass, and therefore the sensitivity approaches zero asymptotically. This behavior is also reflected in the electrical transfer impedance. It suggests the possibility of accelerating artificially this decay in the measured frequency range, provided that the upper frequency is well above the resonance frequency of the microphones. This can be done with a low-pass filter. The low-pass filtered electrical transfer impedance, $Z_{e, 12, L}(f)$ is defined by

$$
Z_{e, 12, L}(f)=Z_{e, 12, E}(f) \cdot L(f),
$$

where $L(f)$ is the low-pass filter. This filter should not alter the modulus of the electrical transfer impedance in the frequency range of concern, and therefore, ideally, it should have a value of unity in the interval $\left[0, f_{f}\right]$ and zero in the rest of the whole frequency range. However, an ideal filter with these characteristics will introduce a noncausality problem on the impulse response; therefore, a causal filter should be applied instead. Such a filter, a linear-phase FIR filter for instance, can be developed by means of the techniques described in several digital signal-processing textbooks, for example in Ref. 18.

\section{B. Time-selective window}

Once the missing portions of the electrical transfer impedance have been completed, the inverse Fourier transform is applied and the impulse response, $z_{e, 12}(t)$, is obtained. The impulse response contains information about the direct wave between the two microphones, the standing wave between the microphones and the reflections from the walls of the anechoic room. In order to remove the last two effects, a time-selective window, $w(t)$, can be applied. This timeselective window must not modify the direct wave between the microphones, and must completely eliminate the reflections, which can be considered as attenuated, delayed replicas of the direct wave. Therefore, the time-selective window should have a value of unity in the time interval where the impulse response contains the direct wave, and zero everywhere else. The first choice may be a rectangular window. However, as this window has high sidelobes, it is expected that it will introduce some ripple in the frequency response caused by cutting abruptly the impulse response where it has not decayed completely to zero. This effect can be reduced by applying a smoothing function on the extremes of the time window. A window with smooth extremes can be generated by convolving the rectangular window with a smoothing function. Although the extremes of the window can be considerably smoothed by the convolution, the height of the first sidelobe is approximately the same as that of the rectangular window. This is a natural consequence of the generation and form of the window itself. One of the convolved windows is the Tukey window ${ }^{19}$

$$
w_{\text {Tukey }}(n)= \begin{cases}1 & 0 \leqslant|n| \leqslant(1+\alpha) N / 2 \\ \frac{1}{2}\left\{1+\cos \left[\pi \frac{n-(1+\alpha) N / 2}{(1-\alpha) N / 2}\right]\right. & (1+\alpha) N / 2 \leqslant|n| \leqslant N \\ & \text { elsewhere, }\end{cases}
$$

where $\alpha$ is the smoothing portion of the window, and $N$ is the length of the window. The convolution procedure can also be applied for other smoothing functions such as those related to Kaiser or Blackman windows. ${ }^{19}$

\section{CALCULATION OF THE SENSITIVITY AND OTHER PARAMETERS}

Once the undesired reflections on the electrical transfer impedance have been removed by the procedure described above, it is possible to use this cleaned function for the calculation of the sensitivity of the microphones and other associated parameters.

The free-field sensitivity of a condenser microphone can be obtained from Eq. (1). This equation contains the product of the free-field sensitivities of the two microphones. It provides an equation with two unknowns. If a third microphone is coupled successively with the other two microphones, a set of three equations with three unknowns is obtained. Then, the sensitivity of each microphone can be obtained in absolute terms. Solving the system of equations, the sensitivity of the first microphone is given in Ref. 12 as

$$
M_{f, 1}=\frac{2}{\rho f} \frac{d_{12} d_{13}}{d_{23}} \frac{Z_{e, 12} Z_{e, 13}}{Z_{e, 23}} \exp \left[\gamma\left(d_{12}+d_{13}-d_{23}\right)\right] .
$$

Similar expressions can be obtained for the other two microphones. This equation is used for calculating the free-field sensitivity after cleaning the electrical transfer impedance functions. Also, it can be proved (see Ref. 12) that the freefield sensitivity can be expressed as a function of the product of the pressure sensitivity (where the diaphragm of the microphone is subjected to a uniform pressure), a diffraction factor $S(f, \theta)$, and the ratio of the impedance of the micro- 
phone, $Z_{a}$, to the radiation impedance $Z_{a, r}$, as follows:

$$
M_{f}=M_{p} \frac{Z_{a}}{Z_{a}+Z_{a, r}} S(f, \theta) .
$$

The latter two factors can be considered as a single one, and renamed as the free-field factor, which is the ratio of the free-field sensitivity to the pressure sensitivity. This quantity is of great practical utility, and it is widely used in field measurements in its logarithmic form and known as the freefield correction. It provides a parameter that is easier to compare with experimental and theoretical results obtained elsewhere. Also, as the most significant effect shown in Eq. (9) is the diffraction factor, it is expected that the free-field correction should be the nearly the same for all microphones with the same geometrical configuration and acoustical impedance.

It can be seen that in Eq. (8) there is a parameter with a significant influence on the final free-field sensitivity. This parameter is the true acoustic distance between the microphones. The true acoustic distance depends on the acoustic centers of the two microphones as defined in Ref. 12.

The experimental determination of the acoustic center can be carried out using simple linear regression techniques if measurements are made at several distances. This procedure can be used when there is confidence in the characteristics of the air in the environment where the measurements are made.

\section{COMPUTER MODELING OF THE ELECTRICAL TRANSFER IMPEDANCE}

A computer model based on Eq. (1) has been developed using MATLAB. The product of sensitivities in the electrical transfer impedance is generated by making use of the lumped-parameter model of the microphones and a typical free-field correction. ${ }^{20}$ The reflections from the imperfect anechoic chamber and the standing wave between the microphones are introduced using the concept of image sources. It should be mentioned that Delany and Bazley ${ }^{21}$ have pointed out that the total field at any point inside the enclosure should be calculated by summing the six waves reflected from the walls and the direct wave from the source with due regard of the relative phase of these waves, and that their relative phase may change as a function of the impedance of the walls. This should give a better agreement between the calculated and measured interference patterns. On the other hand, the walls of the anechoic chamber are formed by a number of wedges that will certainly modify the shape of the reflected wave in an unpredictable manner. However, in this case it is not very important to reproduce the exact shape of the reflections, but to indicate the approximate time instant when they occur. This can be achieved with the use of the image source theory. The cleaning procedure is applied to this simulated electrical transfer impedance in order to evaluate the effect of the procedure as well as of the signalprocessing parameters used to generate it. The selection of such parameters is described below.

The first aspect to be analyzed is that of the duration of the direct wave between the microphones. This can be esti-
TABLE I. Values of the lumped parameters for condenser microphones LS1P.

\begin{tabular}{ll}
\hline \hline & LS1 (B\&K 4160) \\
\hline Acoustic mass, $m_{a}$ & $345 \mathrm{~kg} \mathrm{~m}^{-4}$ \\
Acoustic compliance, $C_{a}$ & $1.19 \times 10^{-12} \mathrm{~m}^{3} \mathrm{~Pa}^{-1}$ \\
Acoustic resistance, $R_{a}$ & $20 \times 10^{-6} \mathrm{~Pa} \mathrm{~s} \mathrm{~m}^{-3}$ \\
\hline \hline
\end{tabular}

mated by making use of the lumped-parameter model of the microphones and the time constant defined in Eq. (2).

The microphones to be analyzed are laboratory standard microphones: 1-in. laboratory standard microphones, LS1P. The above type corresponds to the microphone Brüel and Kjær model 4160. The results from this study can be extended to other types of microphones as for instance the 1/2in. laboratory standard, LS2P. Typical values given by the manufacturer for the lumped-parameter model of the pressure sensitivity are shown in Table I.

The impulse response will have a peak at the instant corresponding approximately to the time it takes for the sound wave to travel from the transmitter microphone to the receiver. After this peak it will decay exponentially according to the time constant defined in Eq. (2). This constant provides a measure of how rapidly the amplitude of an impulse response of a system of a single degree of freedom decays following an exponential behavior, $e^{-(1 / \tau) t}$, where $\tau$ is the time constant, and $t$ is the time. This can be used to predict the time when a given decay is expected to occur. Thus, for the parameters given in Table I, the time constant is $0.0345 \mathrm{~ms}$. This value indicates a fast decay of the impulse response. Note that Eq. (1) contains a product of two systems of a single degree of freedom. Then, the resulting convolution has a time constant that is two times the time constant of one system. Thus, using the calculated time constant, the direct wave should have decayed to one thousandth of its maximum amplitude about $0.5 \mathrm{~ms}$ after the peak has been reached. However, it is worth mentioning that it is expected that the actual values of the lumped-parameter model may change under free-field conditions, due to the presence of a radiation impedance and the diffraction factor. Such changes may modify the resonance frequency and the quality factor of the microphone.

With the above result it is possible to determine whether the reflections from the walls and the expected standing wave between the microphones will be far enough from the direct wave to avoid truncating it when applying the timeselective window. Consider that the microphones are located at a distance of $30 \mathrm{~cm}$ from each other in the middle of an anechoic room having free-space dimensions of $120 \times 80$ $\times 175 \mathrm{~cm}$. At these conditions, the direct wave between the microphones will have its peak at about $0.87 \mathrm{~ms}$, and the amplitude of the impulse response should decay to one thousandth of its maximum at $1.37 \mathrm{~ms}$. On the other hand, the closest disturbance to the direct wave is the standing wave between the microphones. The time instant where it should appear in the impulse response is about $2.6 \mathrm{~ms}$. The rest of the reflections will come at a later time: first from the lateral walls and then from the ceiling and the floor of the chamber. These last two reflections come at a time of about $5.1 \mathrm{~ms}$, but 
as discussed above the actual reflection point on the walls is unknown, and therefore the times given above may change in the actual measurements.

In order to obtain a realistic impulse response, the frequency response should be sampled using parameters that are in full accordance with the physical characteristics of the microphones. The resonance frequency and the quality factor of the microphones, $Q$, give information about the lowest higher-limit frequency, $f_{\max }$, that must be measured or simulated in order to determine a realistic impulse response. For LS1P microphones, the resonance frequency is about 8.5 $\mathrm{kHz}$, and the quality factor is nearly unity. This implies that the system has a broad resonance. And, at a frequency 1.5 times the resonance frequency, the average power has dropped $50 \%$ or $3 \mathrm{~dB}$ the from the value at the resonance frequency; if a realistic decay of the impulse response is to be achieved, the maximum frequency should not be less than the above limit. It must be also noticed that the Eq. (1) contains the frequency as a factor, which corresponds to a differentiation that clearly emphasizes high frequencies. As it is desirable to have a frequency response whose highfrequency values should have decayed sufficiently, a proper minimum value for $f_{\max }$ should be estimated.

As the maximum frequency defines the size of the time step in the corresponding impulse response, the number of sampled frequency points should be large enough (a) to describe properly the irregularities caused by the standing wave and reflections from the walls, and (b) to include all the primary reflections, i.e., to give a time record that includes such reflections.

The first reflection appears with a frequency periodicity of about $380 \mathrm{~Hz}$, and the latest with a period of about 200 Hz. This implies that in order to sample the disturbances appropriately, the size of the frequency step should not be larger than $100 \mathrm{~Hz}$, and less if possible. This figure may change slightly if the distance between the microphones is increased, as it is in practical situations where it can take values between 20 and $40 \mathrm{~cm}$. This is a minor variation, however, because the time where the reflections appear does not change in a significant manner when the distance between the microphones is in the given interval.

The number of frequency samples is given by dividing $f_{\max }$ by the frequency step. The number of frequency points as well as the sampling frequency can be increased in order to obtain a sampling of the shape of the disturbances. These calculated time parameters have been used as a basis for a number of simulations of the cleaning procedure applied to a simulated electrical transfer impedance that contains reflections from the walls.

The first parameter to be evaluated is the upper frequency, $f_{\max }$. The objective is to provide a suitable value of $f_{\max }$ so as to generate a realistic impulse response that contains all the relevant information required for a proper separation of the direct wave between the microphones. For this purpose, an electrical transfer impedance function between two LS1P microphones located $30 \mathrm{~cm}$ from each other has been generated at four different frequency ranges with maximum frequencies, $f_{\max }$, of $1.5 \cdot f_{\text {res }}, 2 \cdot f_{\text {res }}, 3 \cdot f_{\text {res }}$, and 4 $\cdot f_{\text {res }}$. In all four cases the frequency interval has been di-
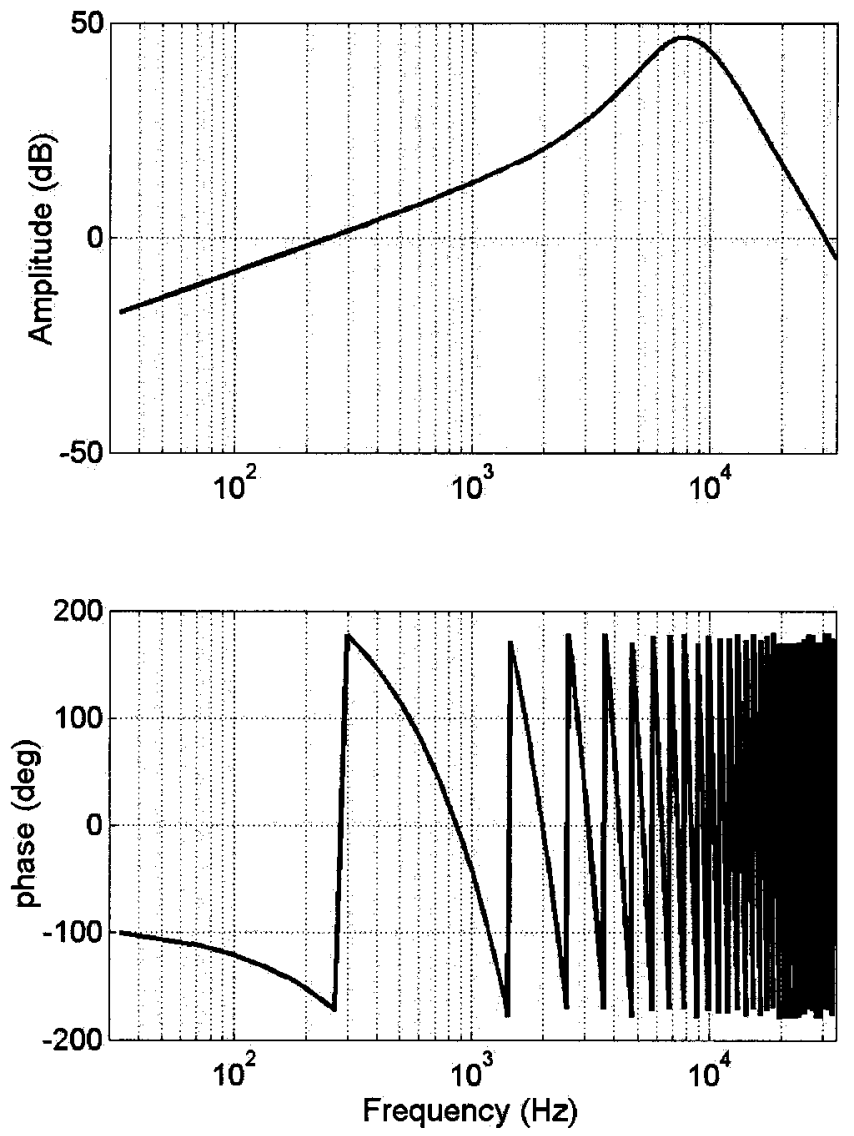

FIG. 4. Simulated electrical transfer impedance function between two LS1P microphones located $30 \mathrm{~cm}$ from each other in a free field. The maximum frequency is $4 \cdot f_{\text {res }}$.

vided into 1024 points. A low-pass frequency window has been applied onto the simulated electrical transfer impedance in order to make it converge to zero at the high-frequency limit. Figure 4 shows a graphic of the electrical transfer impedance function for the last case, $4 \cdot f_{\text {res }}$. Figure 5 shows the Hilbert envelope of the corresponding impulse responses of the four cases.

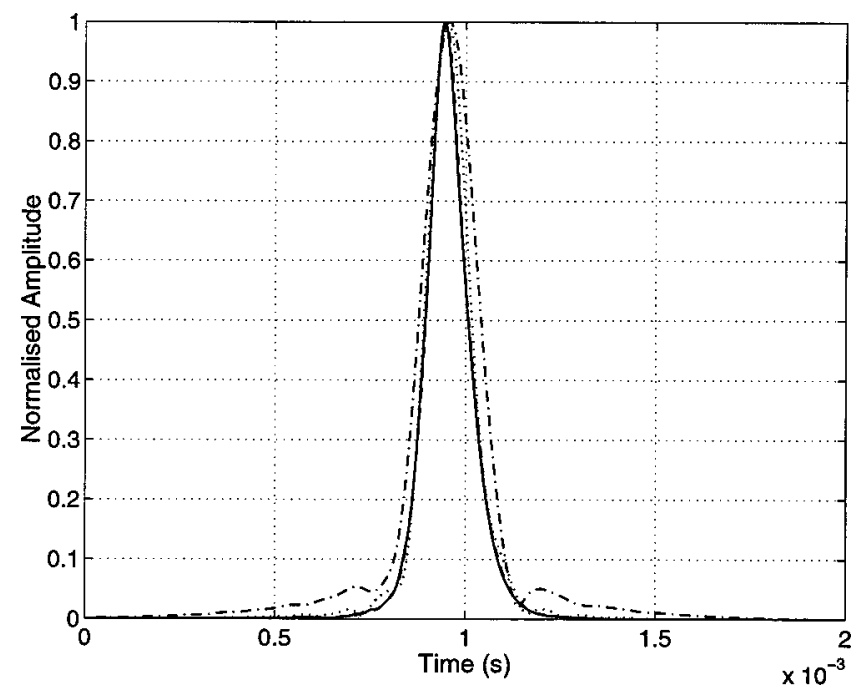

FIG. 5. Hilbert envelope of the impulse response from four simulated electrical transfer impedance functions with maximum frequencies: -4 $\cdot f_{\text {res }} ;---3 \cdot f_{\text {res }} ; \cdots 2 \cdot f_{\text {res }}$, and $-\cdot-1.5 \cdot f_{\text {res }}$. 
(a)
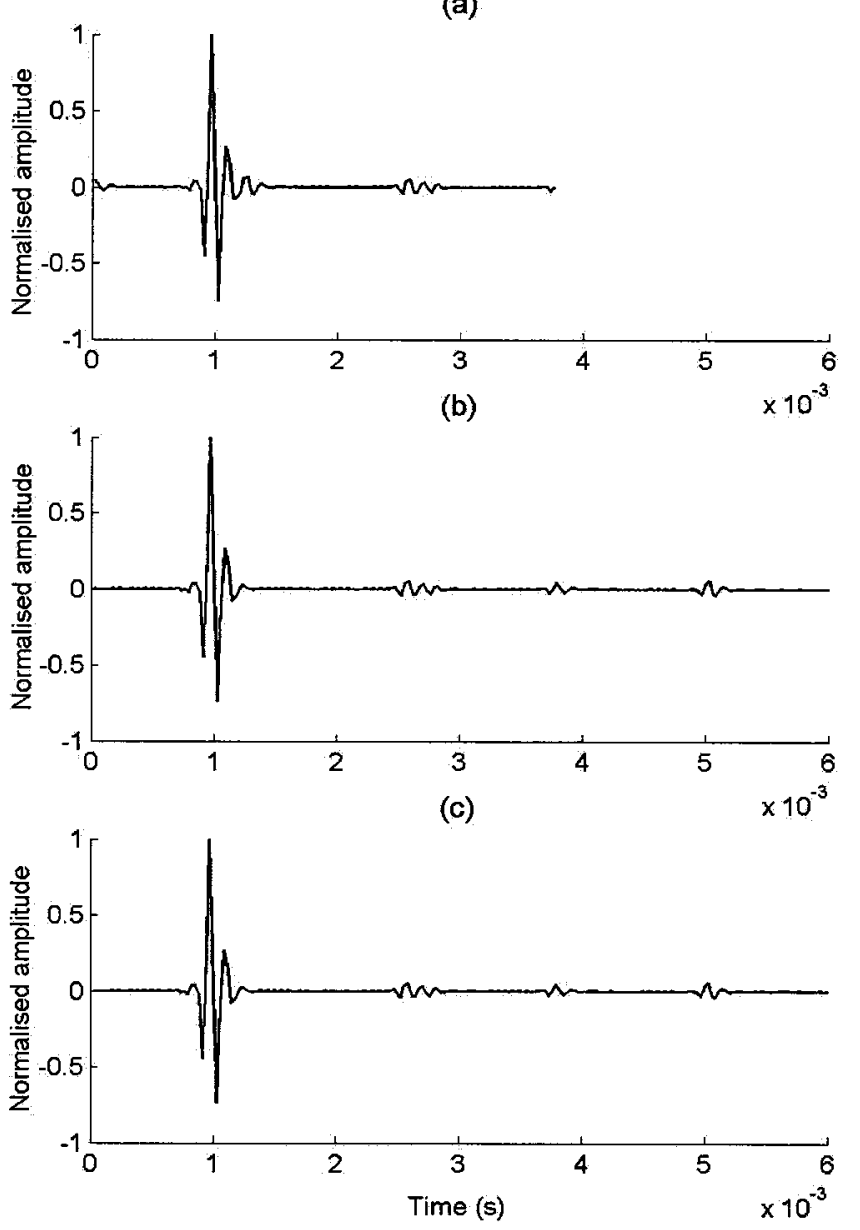

FIG. 6. Impulse response of the simulated electrical transfer impedance function for different sizes of the frequency step: (a) $264 \mathrm{~Hz}$; (b) $132 \mathrm{~Hz}$; and (c) $66 \mathrm{~Hz}$.

As expected, the direct wave between the microphones rises to a maximum after about $0.9 \mathrm{~ms}$. It can be seen that in the case where $f_{\max }=1.5 \cdot f_{\text {res }}$, the impulse response rises and decays slower than under the other three conditions. This has an important consequence if the direct wave is to be isolated from the reflections. Additionally, a small time shift of a fraction of a millisecond can be observed between the peaks of the four cases; the difference tends to converge as the value of $f_{\max }$ increases. This is basically a consequence of the major high-frequency information contained in the frequency responses with a higher $f_{\max }$ and has no important consequence on the overall procedure.

The second parameter to be evaluated is the size of the frequency step. The intention is to find a suitable step size that will represent accurately the effect of the reflections in the impulse response. This is important in this case because the measurements of the electrical transfer impedance are made frequency by frequency, and thus the number of frequency steps should be limited. The simulated electrical transfer impedance corresponds to the case of two LS1P microphones located at a distance of $30 \mathrm{~cm}$ from each other. The upper frequency $f_{\max }$ is chosen to be two times the resonance frequency of the microphones. Artificial reflections have been added by introducing mirror sources. Three cases have been studied, dividing the frequency interval into (a)

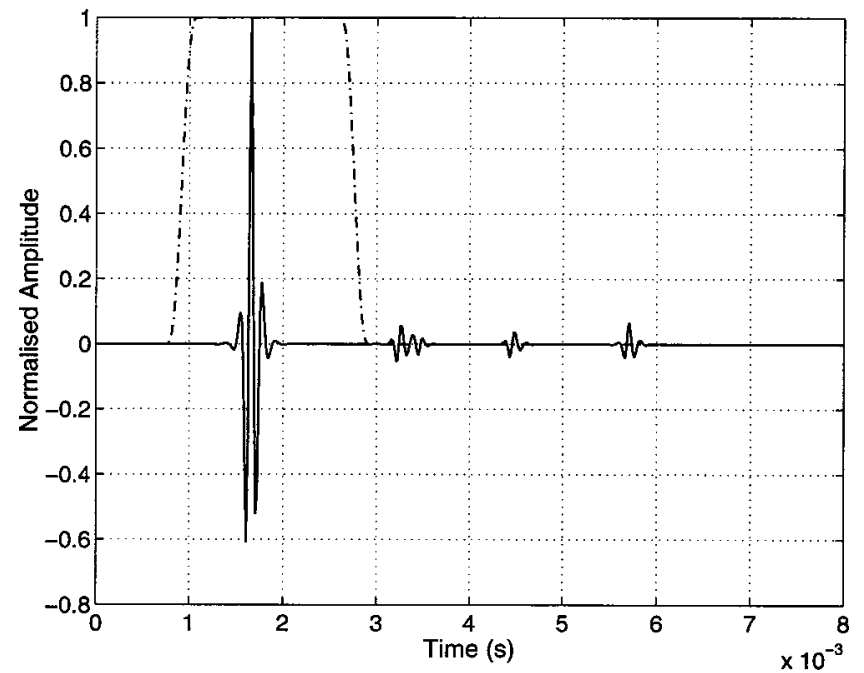

FIG. 7. Impulse response of the simulated electrical transfer impedance function and the time-selective window: —— impulse response, and -·time-selective window.

64; (b) 128; and (c) 256 frequency steps that corresponds to frequency steps of 264,132, and $66 \mathrm{~Hz}$, respectively. The impulse responses corresponding to the three cases are shown in Fig. 6.

It can be seen that the step size of the frequency response is an important parameter. As expected, the large size of the frequency step in case (a) leads to a subsampling of the disturbances caused by the latest of the reflections. This can be seen in Fig. 6(a), where the corresponding impulse response is not long enough to include a portion of the reflections, and even at zero time a portion of the last reflection is present. This may be caused by an aliasing effect. Case (b) describes a situation that is on the limit of aliasing, but it contains all the reflections. Finally, case (c) is a frequency interval short enough to avoid any aliasing. From the above results it can be concluded that it is possible to carry out a simulation of the cleaning procedure of the electrical transfer impedance describing accurately its impulse response. It is intended to assess experimental measurements with the simulation results, and therefore sampling parameters derived from the above methodology that are compatible with those used in the experimental setup are to be used in the following.

The electrical transfer impedance between two LS1P microphones has been generated by using 993 frequency steps in the frequency range from 900 to $30690 \mathrm{~Hz}$, i.e., with a frequency step of $30 \mathrm{~Hz}$. Artificial reflections have been introduced. Background noise has been introduced by adding a random variation to the simulated electrical transfer impedance of the same level to that observed in the measurement setup. The missing portion of the contaminated electrical transfer impedance is filled with values of a reflectionless electrical transfer impedance. Then, a low-pass filter is applied to the frequency response. Thereafter, the impulse response is obtained, and a Tukey window [Eq. (7)] is applied. These are shown in Fig. 7. The peak of the impulse response is expected to occur at $0.87 \mathrm{~ms}$; however, the linear-phase characteristic of the FIR low-pass filter introduces the additional delay that is observed in Fig. 7. 


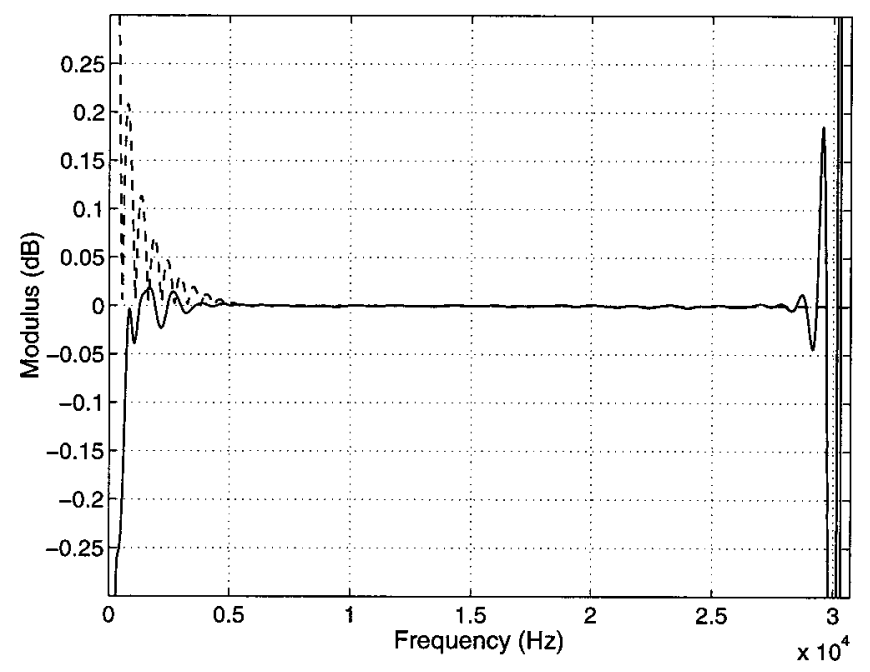

FIG. 8. Simulated difference between the cleaned and an ideal electrical transfer impedance function as a measure of the global effect of the cleaning procedure on the accuracy of the electrical transfer impedance. residual; --- spectrum of the time-selective window.

The most important outcome of this simulation study is the effect caused by the application of the cleaning procedure described in Eq. (3). For this purpose, the cleaned electrical transfer impedance is compared with an ideal reflectionless electrical transfer impedance; the overall effect of the procedure is defined as the difference between the two quantities. This result is to be used for assessing the accuracy improvements caused by the removal of the reflections and any deviation introduced by the procedure. Figure 8 shows the difference between the cleaned electrical transfer impedance and the reflectionless electrical transfer impedance. It can be seen that major differences are present at the extremes of the residual function. These differences are apparently caused by the truncation of the impulse response at an instant when it has not decayed sufficiently. The ripple on the residual is explained by the fact that the time-selective window has a short duration compared with the total length of the impulse response. Thus, its Fourier transform has high and wide sidelobes; it can be seen at the residual function that the frequency of the distortions at the extremes coincides with that present in the Fourier transform of the time window, whose modulus is also shown in Fig. 8.

Another effect that can be observed is that at the patching frequency, $f_{0}$, there is a discontinuity of amplitude and slope between the ideal and the contaminated electrical transfer impedance functions. This is caused by the fact that the ideal value of the frequency response at that frequency may coincide with a maximum or a minimum of the contaminated frequency response. Though this may introduce an additional harmonic distortion, this may not be significant because the patching level is normally $40 \mathrm{~dB}$ lower than the maximum value of the frequency response.

All in all, it can be concluded that the most significant deviations introduced by the cleaning procedure are caused by the time-selective window. The frequency range where the time window introduces the maximum levels of distortion can be determined from its frequency spectrum by evaluating the width and height of the main and secondary
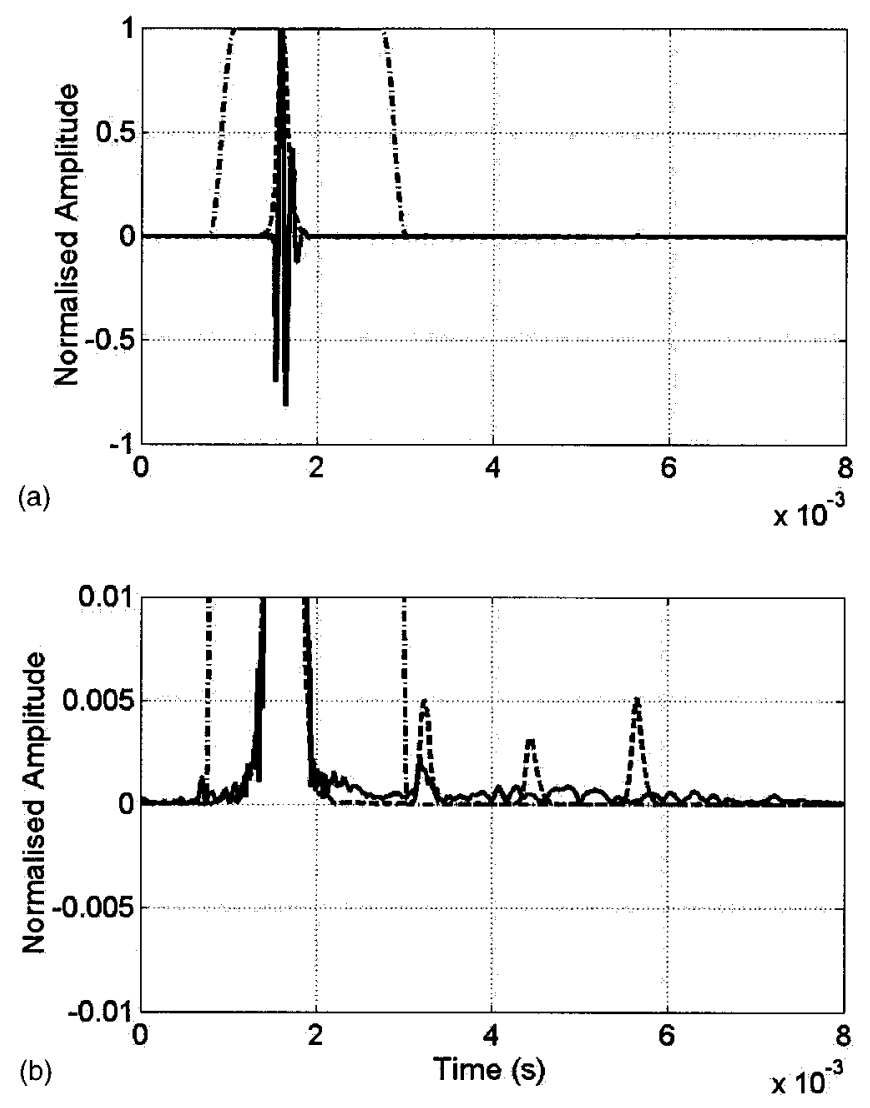

FIG. 9. Impulse response obtained from experimental data. (a) is the normalized amplitude and the time window: —— Impulse response, --- Hilbert envelope of the ideal impulse response; and -.- time window. (b) Hilbert envelope of the measured response, --- Hilbert envelope of the ideal impulse response; and --- time window.

lobes. Thus, the accuracy remains unchanged in the frequency range where the distortion caused by the timeselective window can be considered as negligible. For metrological purposes, this can be set to be compared with a given uncertainty level.

\section{EXPERIMENTAL RESULTS}

In this section, the cleaning procedures have been applied on experimental data from LS1P microphones. The experimental setup consists of three LS1P standard microphones, B\&K 4160, placed in a small anechoic room with free space dimensions of $120 \times 80 \times 175 \mathrm{~cm}$. The measurement of the frequency response is made using the so-called steady-state response mode of the analyzer, B\&K 2012. The analyzer is connected to a reciprocity apparatus that measures the voltage on the terminals of the receiver microphone and the current through the terminals of the transmitter microphone.

Figure 9 shows the impulse response of an electrical transfer impedance between two microphones located at a distance of $28 \mathrm{~cm}$ from each other. It can be seen that the measured impulse response and the ideal impulse response have very similar characteristics in terms of duration and decay time, thus validating the simulation results. Also, the position of the first reflection which is caused by the standing wave between the microphones coincides in the two cases. 


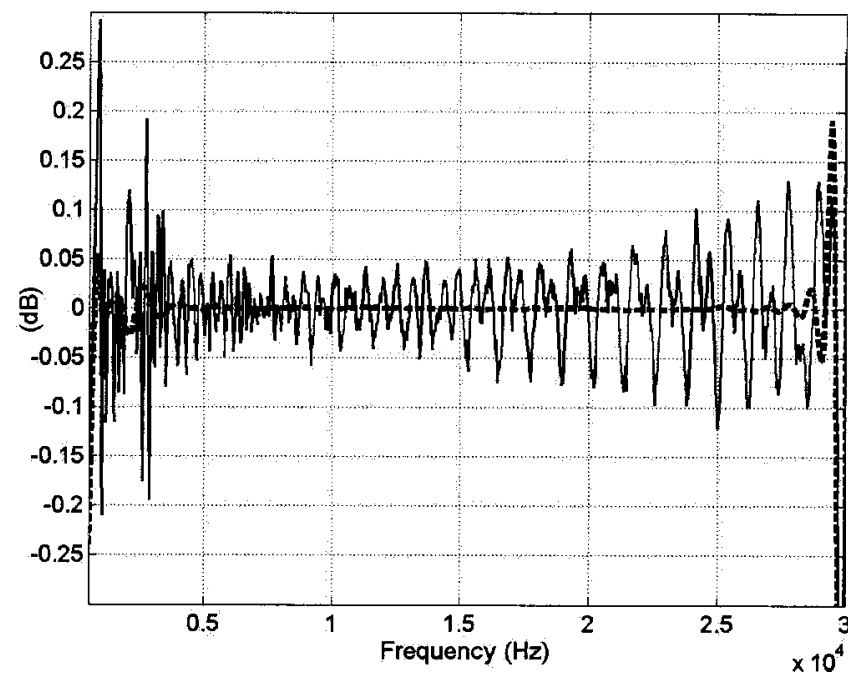

FIG. 10. Difference between the cleaned and the raw experimental electrical transfer impedance function compared with the difference between the cleaned and ideal electrical transfer impedance functions obtained from the simulations. — Difference between cleaned and raw, --- difference between ideal and cleaned.

However, the reflections from the walls appear to come later in the measured data; this may be a consequence of the selection of the reflection point at the wall, which in the simulation is considered to be the tip of the wedges. A more realistic simulation should consider the complicated reflections coming from the wedges.

In order to assess the effectiveness of the technique for removing the reflections, it is necessary to compare the size of the removed disturbances with the size of the distortion introduced by the cleaning procedure (shown in Fig. 8). In Fig. 10 the difference between the cleaned and the measured electrical transfer impedance function for the same case is shown. In Fig. 11 the result of the acoustic centers determined from the cleaned and raw measurements are shown. The acoustic centers were obtained using a linear regression procedure on a set of measurements of the electrical transfer

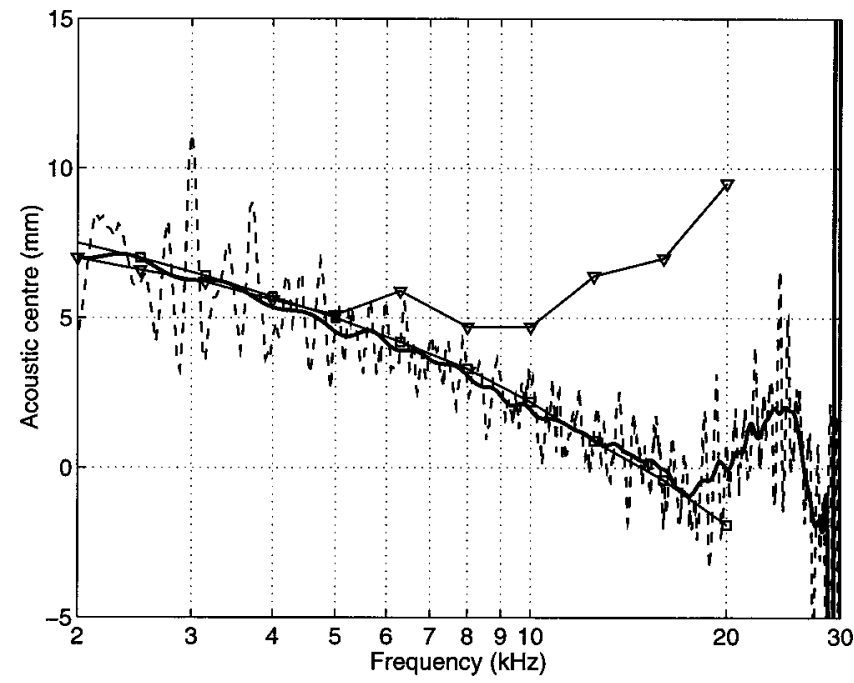

FIG. 11. Position of the measured acoustic centers of three LS1P condenser microphones: — Average of the cleaned for three microphones, --raw, - $\square$ - IEC standard (Ref. 12), and - $\nabla$ - Rasmussen (Ref. 11).

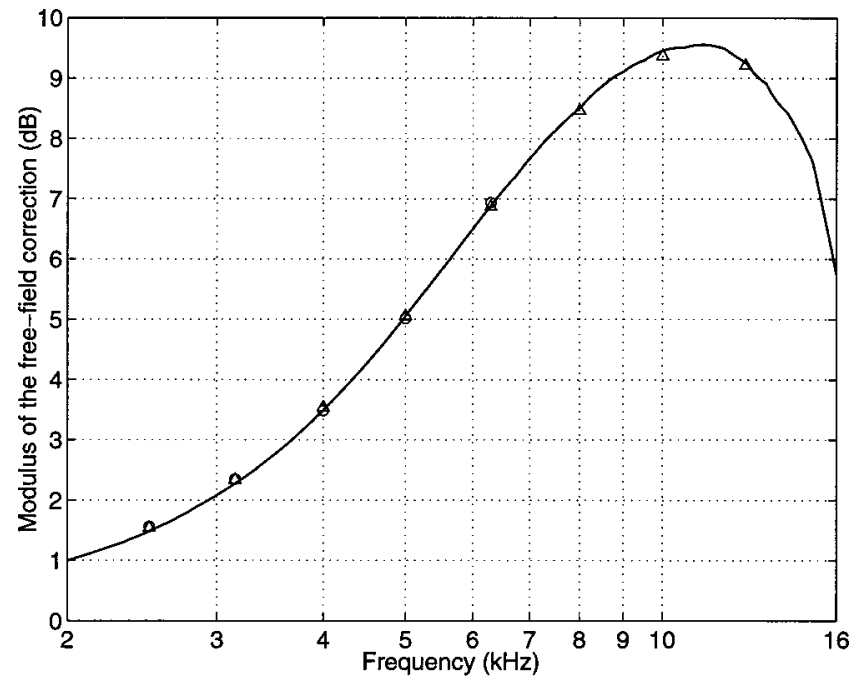

FIG. 12. Experimental free-field correction for LS1P condenser microphones. - Average value for three microphones, $\bigcirc$ Juhl (Ref. 22), and $\triangle$ IEC655 standard (Ref. 20).

impedance at different distances. These results are compared with data provided by the Refs. 11, 12, and 22. It is apparent that the uncleaned or raw measurements give large variations of the acoustic centers. These deviations are not inherent to the acoustic centers but a consequence of the interference patterns of the direct and reflected waves. The approximation obtained using the cleaned electrical transfer impedances appears to follow the values given for comparison better, although it diverges at frequencies above $10 \mathrm{kHz}$. These deviations could be caused by the fact that the acoustic centers are strongly dependent on the velocity distribution of the diaphragm, and around and above the resonance frequency very little can be assumed about the behavior of this quantity.

Another fact that is worth mentioning is the difference between the cleaned acoustic centers and the values given in Ref. 11. The present measurements and those of Ref. 11 were carried out using the same facilities. At the time when this report was written, it was suspected that the difference might be due to an anomalous air absorption inside the anechoic chamber. In the course of the investigation, a number of measurements were made while the door of the small anechoic chamber was open. The results obtained were similar to those given in the standard. ${ }^{12}$ After a series of additional tests, a ventilation system was introduced in the chamber. The results presented here correspond to the new condition, which proves that the air inside the chamber was causing the divergence. Thus, application of the cleaning technique has helped to solve some discrepancies observed between the theory and experimental measurements. Additionally, it shows the importance of verifying that the physical properties of the air inside the anechoic environment where free-field measurements are to be made are those of standard air.

Figure 12 shows the average free-field correction of the three microphones calibrated and values given in Ref. 22 and the standard. ${ }^{20}$ The results shown in Fig. 12 are in good agreement with the previously published data. Unfortunately, because there is not much information available for the high- 
frequency range (above $10 \mathrm{kHz}$ ), the obtained results cannot be compared with another reference.

\section{CONCLUSIONS}

A time-selective procedure has been developed and tested using computer simulations and experimental data. The procedure has proved to remove the imperfections on the electrical transfer impedance caused by reflections effectively. It makes it possible to reduce the uncertainty of the free-field sensitivity.

The acoustic centers of the microphones have been determined experimentally with improved accuracy over an extended frequency range that has not been covered before. The determined values show good agreement with previously published values at frequencies below and around the resonance frequency. The high-frequency values provide a new reference for further analysis. Additionally, the obtained values have helped to solve some discrepancies observed in previously published works.

The free-field sensitivity was calculated from the cleaned electrical transfer impedances using the experimental acoustic centers. The low-frequency values of free-field sensitivity and, thus, the free-field correction are also in agreement with previously published data. The accuracy of the calculated sensitivity has been improved significantly in an extended frequency range not covered in previous work. The time-selective technique makes it possible to remove reflection artifacts from the data, thereby effectively obtaining free-field calibration conditions.

\section{ACKNOWLEDGMENTS}

This research project is carried out with the support of the Consejo Nacional de Ciencia y Tecnología (CONACYT) and the Centro Nacional de Metrología (CENAM) of México.

\footnotetext{
${ }^{1}$ W. R. MacLean, "Absolute measurement of sound without a primary standard," J. Acoust. Soc. Am. 12, 140-146 (1940).

${ }^{2}$ W. Wathen-Dunn, "On the reciprocity free-field calibration of microphones," J. Acoust. Soc. Am. 21(5), 542-546 (1949).

${ }^{3}$ I. Rudnick and M. N. Stein, "Reciprocity free field calibration of micro-
}

phones to $100 \mathrm{Kc}$ in air,” J. Acoust. Soc. Am. 20(6), 818-825 (1948).

${ }^{4}$ A. L. DiMattia and F. M. Wiener, "On the absolute pressure calibration of condenser microphones by the reciprocity method," J. Acoust. Soc. Am. 18(2), 341-344 (1946).

${ }^{5}$ R. L. Terry and R. B. Watson, "Pulse technique for the reciprocity calibration of microphones," J. Acoust. Soc. Am. 23(6), 684-685 (1951).

${ }^{6}$ A. F. Niemoeller, "Reciprocity calibration of condenser microphones in the time domain," J. Acoust. Soc. Am. 33(12), 1712-1719 (1961).

${ }^{7}$ D. L. H. Gibbings and A. V. Gibson, "Free-field reciprocity calibration of capacitor microphones at frequencies from $19.95 \mathrm{kHz}$ to $316.2 \mathrm{~Hz}$," Metrologia 20, 85-94 (1984).

${ }^{8}$ E. W. Burnett and V. Nedzelnitsky, "Free-field reciprocity calibration of microphones," J. Res. Natl. Bur. Stand. 92(2), 129-151 (1987).

${ }^{9}$ J.-N. Durocher, "Etalonnage des microphones à condensateur en champ libre," J. Acoust. 2, 431-436 (1989).

${ }^{10}$ R. Barham, "Free-field reciprocity calibration of laboratory standard microphones," Ph.D thesis, ISVR, University of Southampton, 1995.

${ }^{11}$ K. Rasmussen and E. Sanderman Olsen, "Intercomparison on free-field calibration of microphones," The Acoustics Laboratory, Technical University of Denmark, Report PL-07 (1993).

${ }^{12}$ IEC 61094-3 (1995-11), "Measurement microphone Part 3: Primary method for free-field calibration of laboratory standard microphones by the reciprocity technique" (1995).

${ }^{13}$ J. M. Lambert and J. N. Durocher, "Analyse des perturbations acoustiques lors de l'etalonnage en champ libre des microphones étalons à condesateurs dits d'un pouce par la technique de la réciprocité" (in French), Laboratoire National D'Essais (1989)

${ }^{14}$ J. S. Blem, "Tidsselektiv fritfeltskalibrering af kondensatormikrofoner" (in Danish), Master thesis, The Acoustics Laboratory, Technical University of Denmark (1992).

${ }^{15}$ M. Vorländer and H. Bietz, "Novel broad band reciprocity technique for simultaneous free-field and diffuse-field microphone calibration," Acustica 80, 365-377 (1994).

${ }^{16} \mathrm{~V}$. Tarnow, "Thermal noise in microphones and preamplifiers," Brüel \& Kjær Technical Review, 3-14 (1972) (3).

${ }^{17} \mathrm{~K}$. Rasmussen, "The static pressure and temperature coefficients of laboratory standard microphones," Metrologia 36, 265-273 (1999).

${ }^{18}$ J. G. Proakis and D. G. Manolakis, Digital Signal Processing. Principles, Algorithms and Applications, 3rd ed. (Prentice-Hall, Englewood Cliffs, NJ, 1996).

${ }^{19}$ F. J. Harris, "On the use of windows for harmonic analysis with the discrete Fourier Transform,” Proc. IEEE 66(1), 51-83 (1978).

${ }^{20}$ IEC International Standard 655, "Values for the difference between freefield and pressure sensitivity levels for one-inch standard condenser microphones" (1979).

${ }^{21}$ M. E. Delany and E. N. Bazley, "A note on the sound field due to a point source inside and absorbent-lined enclosure," J. Sound Vib. 14(2), 151157 (1971).

${ }^{22}$ P. Juhl, "A numerical investigation of standard condenser microphones," J. Sound Vib. 177(4), 433-446 (1994). 